\title{
Bildung, chancengleiche Konkurrenz und gleiche gesellschaftliche Teilhabe
}

Seit den ersten PISA-Ergebnissen" (2000) haben alle Parteien das Thema „Chancengleichheit“ wiederentdeckt und mit neuartigen Akzenten wie „Bildungsgerechtigkeit“ oder „Chancengerechtigkeit“ versehen. Dabei droht das Ziel in den Hintergrund zu treten, tatsächlich gleiche Befähigungen als Voraussetzung für ein selbstbestimmtes Leben zu erreichen. Der Beitrag argumentiert, dass ein solcher Anspruch an das öffentliche Bildungssystem durchaus realisierbar ist, derzeit aber Vielen institutionell verwehrt wird. ${ }^{2}$

\section{1 \\ Einleitung}

Bildung erlebt in der Politik derzeit eine programmatische Blüte wie zuletzt in den 1960er und 70er Jahren. Es gibt kaum ein Problem, das nicht mithilfe von Bildungsund Lernprozessen für Jung und Alt einer Lösung nähergebracht werden soll. In der rohstoffarmen Bundesrepublik Deutschland mutieren die kleinen grauen Zellen in den Köpfen der Menschen, namentlich der jungen, zum „Rohstoff Bildung“, der unbedingt zu mehren ist, damit das Wirtschaftswachstum erhalten und das hohe Lebensniveau gesichert werden kann.

Unabhängig von dieser ökonomischen Betrachtung von Bildung wird auch ihre Rolle in einer ungleichen Sozialstruktur erörtert. Die fehlende Durchlässigkeit des deutschen typisierten Systems von Allgemein-, Berufs- und Weiterbildung und die hohe Abhängigkeit des Bildungserfolgs von der sozialen Herkunft der Schüler und Studierenden verweisen auf eine vertikal stark strukturierte, wenig egalitäre und immobile deutsche Gesellschaft (Pollak 2010). Diesem Sachverhalt stehen die Forderungen nach chancengleicher Bildung und gleicher gesellschaftlicher Teilhabe gegenüber. Der vorliegende Beitrag stellt sich die Frage, wie diese beiden gesellschaftspolitischen Ziele genauer beschrieben werden können und wie sie im deutschen Bildungssystem institutionell verankert sind. Sie werden in der öffentlichen Debatte unter dem Oberziel „Chancengleichheit“ häufig in einem Atemzug genannt, darunter verbergen sich aber wichtige Unterschiede. Im Folgenden wird die These vertreten, dass die Struktur des deutschen Bildungssystems die ohnehin vorhandene Spannung zwischen diesen beiden Zielen verschärft.

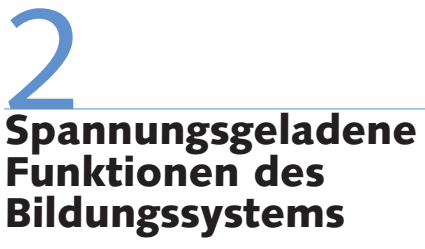

Eine zentrale Funktion des Bildungssystems in kapitalistischen, marktförmigen Gesellschaften besteht darin, für eine Struktur ungleicher Berufe und entsprechender Arbeitsmärkte ungleiche Qualifikationen, Abschlüsse und Berechtigungen bereitzustellen. Dies erfolgt maßgeblich, aber nicht ausschließlich, in einem umfassenden System der ununterbrochenen Erstausbildung, das Lernprozesse vom Kindes- bis ins frühe Erwachsenenalter organisiert. Bildung und Ausbildung vollziehen sich als außerordentlich lang anhaltende Konkurrenz um knappe, privilegierende Ausbildungen für knappe privilegierte Berufe. Leistungsprüfungen regeln das Voranschreiten in der Bildungslaufbahn und den Übergang in das Beschäftigungssystem. Die Konkurrenz soll leistungsbestimmt, offen und fair in dem Sinne sein, dass alle Teilnehmer in etwa gleiche Startbedingungen haben und der Wettbewerb selbst nicht zugunsten bestimmter Gruppen verzerrt ist. Dann ist "Chancengleichheit" im Sinne von Leistungsgerechtigkeit in einem Bildungswettbewerb gegeben, dessen Ergebnisse durchaus ungleich sein können.

Das Ziel gleicher gesellschaftlicher Teilhabe im Sinne gleicher Lebenschancen unterscheidet sich von der Chancengleichheit in einem Bildungswettbewerb in dreierlei Hinsicht:

- es reicht biografisch weit über Bildungsabschlüsse hinaus und bezieht sich auf den gesamten Lebensverlauf;

- „Lebenschancen“ sind inhaltlich weiter gefasst als berufsbezogene Bildungschancen;
- und der Zugang zu ihnen unterliegt keinem Leistungswettbewerb.

Gleiche „Chancen“ heißt nach dem Teilhabekonzept und bezogen auf Bildung: Ausstattung mit einem Standard an Bildung, der jedes Gesellschaftsmitglied zu einem selbstbestimmten und befriedigenden Leben befähigen soll. Dazu dient nicht nur die Erstausbildung, sondern auch die den Lebenslaufbegleitende Weiterbildung. Zwar sind Berufe eine zentrale Grundlage für die Einbindung in die gesellschaftliche Arbeitsteilung (vgl. hierzu Kronauer 2010, S. 14ff.); aber gesellschaftliche Teilhabe umfasst mehr als berufliche Integration. Sie bedeutet Teilhabe am politischen, kulturellen, freizeitlichen und geselligen Leben weit über den Beruf hinaus (Autorengruppe Bildungsberichterstattung

Die PISA-Studien der OECD sind internationale Schulleistungsuntersuchungen, die seit dem Jahr 2000 in dreijährigem Turnus in den meisten Mitgliedstaaten der OECD und einer zunehmenden Anzahl von Partnerstaaten durchgeführt werden und die zum Ziel haben, alltags- und berufsrelevante Kenntnisse und Fähigkeiten 15-jähriger zu messen. Das Akronym PISA wird in den beiden Amtssprachen der OECD unterschiedlich aufgelöst: englisch als Programme for International Student Assessment (Programm zur internationalen Schülerbewertung) und französisch als Programme international pour le suivi des acquis des élèves (Internationales Programm zur Mitverfolgung des von Schülern Erreichten)“, (http://de.wikipedia. org/wiki/PISA-Studien).

2 Für sehr hilfreiche Kommentare zum Text danke ich Irene Becker, Martin Kronauer und Günther Schmid.

Wulf Hopf, Prof. Dr., war vor seinem Ruhestand am Pädagogischen Seminar der Universität Göttingen tätig. Arbeitsschwerpunkte: Bildung und soziale Ungleichheit, Methoden, politische Sozialisation.

e-mail:whopf@uni-goettingen.de 
2010, S. 201ff.). Das schließt auch die Sicherung gegenüber Risiken ein, die im Verlauf des Lebens auftreten können und die die gesellschaftliche Teilhabe erschweren (z.B. Krankheit, instabile Beschäftigung und Arbeitslosigkeit, Altersarmut). Das Konzept einer präventiven „Bildungssozialpolitik“, die die konventionellen sozialpolitischen Maßnahmen ergänzt, richtet sich auf diesen Zusammenhang (vgl. z.B. Allmendinger/Leibfried 2002).

Damit stellt sich die Frage, wie diese beiden, in Inhalt und Reichweite verschiedenen gesellschaftspolitischen Ziele in einem konkreten Bildungssystem verankert und gewichtet sind. Dazu sollen in einem ersten Schritt die beiden Ziele näher charakterisiert werden (Abschnitt 2.1 und 2.2). In einem nächsten Schritt (Abschnitt 3) wird empirisch gefragt, inwieweit im deutschen allgemeinbildenden Schulsystem Startchancengleichheit für einen Bildungswettbewerb und Gleichheit von Bedingungen gesellschaftlicher Teilhabe realisiert sind (3.1). Im Anschluss daran werden institutionelle Besonderheiten des deutschen Schulsystems dargestellt, die eine bestimmte Gewichtung zwischen den beiden gesellschaftspolitischen Zielen bedingen (3.2 und 3.3). Eine Skizze notwendiger bildungspolitischer Veränderungen (Abschnitt 4) schließt den Beitrag ab.

\subsection{CHANCENGLEICHHEIT IM BILDUNGSWETTBEWERB}

Wie sieht ein chancengleicher Leistungswettbewerb innerhalb eines Bildungssystems aus, bei dem die Schüler und Schülerinnen ihre Fähigkeiten und Interessen unabhängig von ihrer sozialen Herkunft entfalten können? Dazu kann man sich ein vereinfachtes Modell vorstellen. Es unterscheidet eine gemeinsame Primarbildung von einer Sekundarbildung, die in zwei Schultypen erfolgen kann: einem , hohen $\mathrm{H}^{\text {“ }}$ und einem „niedrigen N“. Beide Schultypen unterscheiden sich hinsichtlich Inhalt und Dauer der Ausbildung und führen zu unterschiedlichen Abschlüssen für ungleiche Berufe. Der Übergang in die unterschiedlich privilegierenden Sekundarschulen $\mathrm{H}$ und $\mathrm{N}$ wird durch eine Kombination von freier Wahl der Sekundarschulen und Leistung am Ende der Primarbildung geregelt. Da Kompetenzen und Interessen der Kinder für die unterschiedlichen Schulformen $\mathrm{H}$ und $\mathrm{N}$ in dem Übergangsverfahren nicht genau bestimmt werden können und sich zudem noch entwickeln, müssen $\mathrm{H}$ und $\mathrm{N}$ „durchlässig“"zueinander sein, d.h. Wechsel der Schüler in beiden Richtungen erlauben.

Ein chancengleicher Leistungswettbewerb ist in einem solchen vereinfachten Modell unter drei Bedingungen möglich:

(1) In der Phase der Primarbildung müssen gleiche Bildungsergebnisse der Schüler und Schülerinnen erzielt werden, damit der anschließende Wettbewerb unter fairen Startbedingungen ablaufen kann. Da Eltern aus unterschiedlichen Schichten über unterschiedliche Ressourcen für die Bildung ihrer Kinder verfügen und diese entsprechend ihren Möglichkeiten einsetzen, muss die Schule während der Primarbildung kompensatorisch für ressourcenarme Familien tätig werden und die Leistungskonkurrenz von ressourcenreichen Eltern und Schülern in dieser Phase zurückdrängen oder umlenken. Die Notwendigkeit, gleiche Ausgangsbedingungen für einen Leistungswettbewerb zu schaffen, kann man nicht einem Wettbewerb überlassen.

(2) Beim Übergang auf die Schultypen $\mathrm{H}$ und $\mathrm{N}$ sind Leistung und freie Wahl der Ausbildung so zu balancieren, dass im Ergebnis Leistungen entscheiden und nicht die schichtabhängige Wahl der Schultypen (siehe dazu Abschnitt 3.2).

(3) Die jeweiligen Potenziale, in Schultyp $\mathrm{H}$ oder $\mathrm{N}$ überzuwechseln, müssen erkannt, gefördert und voll ausgeschöpft werden.

Das reale deutsche Bildungssystem ist komplizierter als dieses Modell. Im pädagogischen Alltag erfüllen allenfalls herausgehobene kurze Prüfungsphasen die Bedingungen eines von außen ungestörten Leistungswettbewerbs unter den zwangsweise vereinzelten Schülern und Schülerinnen. In den übrigen, weitaus längeren Phasen normalen Unterrichts gehören die Kooperation der Schüler und die mehr oder weniger permanente Einflussnahme von außen - durch die Eltern und die "peers", durch das soziokulturelle Umfeld der Familie-zum Geschehen. Dadurch ist eine säuberliche Trennung von anfänglicher Egalisierung der Startbedingungen und anschließender Freigabe eines von außen unbeeinflussten Leistungswettbewerbs kaum möglich.

Auch die faktische Organisation des Schulsystems verwischt das vereinfachte
Modell des chancengleichen Bildungswettbewerbs. Sie ist in Deutschland bis zum Hochschulabschluss durch mehr vertikale Stufen und horizontale Verzweigungen gekennzeichnet als das vereinfachte ZweiTypen-Modell. Jedes erreichte Bildungsniveau - nicht nur der Abschluss der Grundschule - kann dann als Einschnitt verstanden werden, bis zu dem Chancen egalisierende Bildung zu erfolgen hätte, bevor in der anschließenden Phase eine differenzierende Leistungskonkurrenz erfolgt. Die Forderung nach „proportionaler Chancengleichheit" (Hopf 2010, S. 67ff.) heißt dann: Wenn $40 \%$ aller Eltern von Kindern und Jugendlichen Arbeiter sind, dann sollten auf allen Stufen von Schule und Hochschule auch die entsprechenden Absolventen zu 40 \% aus Arbeiterfamilien kommen. Dies ist bekanntlich nicht der Fall. Zum Beispiel kommen 40 \% aller 19bis unter 25-Jährigen im Jahr 2007 aus Arbeiterfamilien, aber unter den Studienanfängern (Universitäten, Fachhochschulen und Verwaltungsfachhochschulen zusammengenommen) machen sie nur $20 \%$ aus (Autorengruppe Bildungsberichterstattung 2010, S.123).

Bildungskonkurrenz um knappe Berechtigungen (mit all ihren unerwünschten Folgen für das Lernen von Inhalten und für soziale Beziehungen, vgl. Streckeisen et al. 2007) ist also im deutschen allgemeinbildenden Schulsystem fest installiert, aber es handelt sich um eine unvollständige, episodische und sozial verzerrte Konkurrenz, deren Endergebnis allein den Individuen zugeschrieben wird.

\subsection{BILDUNG FÜR GLEICHE GESELLSCHAFTLICHE TEILHABE}

Den Perspektivwechsel hin zu einem außerwettbewerblichen Sinn von Lebenschancen illustriert die international vergleichende Schulleistungsstudie PISA. Sie definiert im Anschluss an die OECD (Organisation für wirtschaftliche Zusammenarbeit und Entwicklung) für 15-Jährige „Basiskompetenzen“, „die in modernen Gesellschaften für eine befriedigende Lebensführung in persönlicher und wirtschaftlicher Hinsicht sowie für eine aktive Teilnahme am gesellschaftlichen Leben notwendig sind“" (Deutsches PISA-Konsortium 2001, S. 16). Solche Basiskompetenzen werden in einer „Grundbildung" vermittelt und umfassen in den internationalen Vergleichsstudien Kompetenzen in Lesen, Mathematik und 
Naturwissenschaften. Die Vorstellung einer solchen Grundbildung kann aber problemlos auf andere Domänen - z.B. politische, fremdsprachliche, ästhetische - erweitert werden. Außerdem schließt sie fächerübergreifende Kompetenzen wie Problemlösen, Kommunikations- und Kooperationsfähigkeit ein.

Damit wird eine Art „zivilisatorische Mindestausstattung" an Bildung für gesellschaftliche Teilhabe umrissen, die Auswirkungen für die Inhalte, die Dauer und die Organisation von Bildungsprozessen haben muss. Im Konzept des innerschulischen, chancengleichen Leistungswettbewerbs kann die Eingangsphase des Kompetenzausgleichs kurz gehalten und die Inhalte können eng begrenzt werden. Es reicht der Selbstbezug auf den tradierten Kanon der Schulfächer (z.B. in Deutsch, Mathematik, einer Fremdsprache und einer Naturwissenschaft). Dagegen sind „Basiskompetenzen“ für das befriedigende Leben in der Gesellschaft und für gesellschaftliche Teilhabe sozial offener, langfristiger und inhaltlich anspruchsvoll definiert. Wenn sie für 15-Jährige, d.h. am Ende der Ausbildung der Sekundarstufe I gleich sein sollen, sind wesentlich stärkere Anstrengungen erforderlich als bei der Schaffung von gleichen Wettbewerbsbedingungen nach Ende der Grundschulzeit.

Die Vorstellung eines Mindeststandards an Bildungskompetenzen für gesellschaftliche Teilhabe und ein befriedigendes Leben als Erwachsener befreit Bildungsprozesse aus der Engführung der leistungsbestimmten Bildungskonkurrenz um Abschlüsse und Berechtigungen. Sie erlaubt es, neue Konzepte sinnvoller Ausbildungsinhalte kreativ zu bestimmen, die nicht sofort mit Schulabschlüssen und Berechtigungen verbunden sein müssen. Zwar bieten Berechtigungen für diejenigen, die sie besitzen, einen gewissen Schutz und vermitteln damit Sicherheit. Sie eröffnen Zugänge für Anschlussausbildungen und Berufe und schließen diejenigen aus der Konkurrenz aus, die nicht über die entsprechende Berechtigung verfügen. Aber der Erwerb von Basiskompetenzen für gesellschaftliche Teilhabe ist ebenfalls ein Mittel, damit das erwachsene Individuum aktiv und bewusst mit Risiken umgeht und ihnen nicht einfach ausgeliefert ist. In dieser Befähigung liegt - jenseits von Berechtigungen - ebenfalls ein Sicherheit vermittelndes Moment.
So unterschiedlich die gleichen Startbedingungen in einem wettbewerblichen Konzept von Chancengleichheit und von gleichen „Basiskompetenzen“ bei einem Konzept gleicher gesellschaftlicher Teilhabe sein mögen - es bleiben gleiche Kompetenzen. In beiden Formen wird auf wirkliche Gleichheit der Ergebnisse gezielt. Das ist deshalb wichtig zu betonen, weil unter der Flagge von „Chancengerechtigkeit“ oder „Bildungsgerechtigkeit“ alle möglichen Revisionen des anspruchsvollen Zieles gleicher Ergebnisse segeln und Gleichheit von vornherein für utopisch erklärt wird.

\section{Empirische Befunde}

\subsection{DIE EGALISIERUNG VON MIN- DESTKOMPETENZEN AM ENDE DER GRUNDSCHULE}

Das Mindestniveau gleicher Startbedingungen für einen Bildungswettbewerb sollte in Deutschland am Ende der Grundschulbildung erreicht sein. $\mathrm{Zu}$ diesem Zeitpunkt sollten herkunftsbedingte Unterschiede der Schüler und Schülerinnen kompensiert und die Basiskompetenzen gleich sein. Ob die Grundschule allen Schülern gleiche Mindestkompetenzen (z.B. in Lesen und Textverständnis, Mathematik, Naturkunde etc.) tatsächlich vermittelt, kann auf zweierlei Weise bestimmt werden: Durch das Ausmaß der Streuung von Kompetenzen um den Mittelwert einer Schulklasse, einer Jahrgangsstufe o.ä. und durch das Ausmaß, in dem Leistungen oder Basiskompetenzen am Ende der Grundschulzeit von der sozialen Herkunft der Schüler und Schülerinnen abhängen.

Beide Maße drücken nicht dasselbe aus. Das erste Maß entspricht noch am ehesten der Alltagssicht von Lehrern, Schülern und Eltern. Sie beziehen Gleichheit und Ungleichheit auf die schulintern sichtbar werdenden Leistungsunterschiede zwischen Schülern „ohne Ansehen der Person“, d.h. unter Aussparung zugeschriebener Merkmale wie Schichtzugehörigkeit, ethnische Zugehörigkeit, Geschlechterstatus etc. Wenn es gelingt, die Divergenz von Schulleistungen in einer Lerngruppe gegen null zu bringen, braucht man nicht mehr nach externen sozialen Bedingungsfaktoren zu fragen. Dann haben alle das gleiche Bil- dungsergebnis erreicht. ${ }^{3}$ Dagegen werden beim zweiten Maß von Bildungschancen die Leistungsunterschiede von vornherein als Differenzen unterschiedlicher externer (gesellschaftlicher) Gruppenbildung verstanden, also z.B. die unterschiedlichen Bildungschancen von Schichten, Einkommensgruppen, Ethnien, Geschlechtergruppen und Stadt-Land-Gruppen.

Im Hinblick auf das erste Maß von Ungleichheit ergibt die international vergleichende Grundschulstudie IGLU (Internationale Grundschul-Lese-Untersuchung), dass in Deutschland ein relativ hohes Kompetenzniveau (Leseleistung) in der vierten Jahrgangsstufe mit einer im internationalen Vergleich relativ geringen Streuung der Leistungen um den Mittelwert verbunden ist. Deutschland gehört zu dem oberen Viertel der Staaten, denen es gelingt, „einen Großteil der Schülerinnen und Schüler auf ein adäquates Leseniveau zu bringen“ (Bos et al. 2007, S. 153).

Auch wenn die Mindestkompetenzen am Ende der Grundschulzeit im internationalen Vergleich relativ wenig streuen, sind Kompetenzunterschiede vorhanden. Inwieweit hängen sie von der sozialen Herkunft ab, verstanden als Schichtzugehörigkeit? Für dieses zweite Maß von Ungleichheit erhoben die Forscherinnen und Forscher von IGLU als einzelne Merkmale des familialen Hintergrundes den Besitz an Büchern in der Familie, die höchste Sozialschichtzugehörigkeit und den höchsten Bildungsabschluss eines Elternteils. Hieraus bildeten sie einen kombinierten Index der sozialen Herkunft und teilten die Befragten nach der Höhe der sozialen Herkunft in vier Quartile (jeweils $25 \%$ der Befragten). Die Differenz im Leseverständnis der Schüler aus dem obersten und dem untersten Quartil der sozialen Herkunft beträgt im Durchschnitt aller Nationen 59 Punkte. Die Bundesrepublik Deutschland liegt mit 67 Punkten über diesem Durchschnitt in einer mittleren Gruppe von Staaten (zwischen 51 und 85 Punkten), von denen sich der deutsche Differenzwert nicht signifikant unterscheidet. Dagegen unterscheidet er sich signifikant von den höheren Differenzwerten einer kleinen Gruppe von Ländern (zwischen 81 und 96 Punkten) und von einer größeren Gruppe von Staaten, in denen die

3 Vgl. zur Verwendung des Streuungsmaßes im finnischen Schulsystem Välijärvi 2008, S. 82ff. 
Differenz zwischen dem obersten Viertel der Schichtungshierarchie und dem untersten Viertel niedriger liegt (zwischen 26 und 59 Punkten) (vgl. Bos et al. 2007, Abb. VIII.8, S. 240). „Offensichtlich gelingt es in Deutschland den vorschulischen Einrichtungen und der Grundschule nicht so gut wie möglicherweise in vielen anderen Staaten, bestehenden sozialen Ungleichheiten kompensierend zu begegnen" (ebd., S. 245). Wirklich gleiche Mindestkompetenzen als Voraussetzungen eines anschließenden Leistungswettbewerbs sind also nicht gegeben. Die herkunftsbedingten Unterschiede werden noch verstärkt, wenn man die Rolle der elterlichen Wahlmöglichkeiten beim Übergang in die Schulen der Sekundarstufe betrachtet.

\subsection{LEISTUNG UND WAHLRECHT IN EINEM TYPENSCHULSYSTEM}

Die Differenzierung der Schüler und Schülerinnen nach Kompetenzen, Leistungen und Berechtigungen erfolgt in Deutschland immer noch über ein historisch überkommenes Typenschulsystem von Gymnasien, Realschulen, Hauptschulen und Schulen für Lernbehinderte. An dieser Typisierung des Sekundarschulwesens hat die Einführung von Gesamtschulen ebenso wenig geändert wie die Tendenz, "Schulen mit mehreren Bildungsgängen" (meistens Haupt- und Realschule) einzurichten oder noch einen Schritt weiter in Richtung eines zweigliedrigen Schulsystems von Gymnasien und integrierten „Sekundarschulen" oder ähnlicher Konzepte zu gehen. ${ }^{4}$ Die Durchlässigkeit zwischen den Schultypen ist nicht sehr hoch; wenn es Wechsel gibt, dann weniger in Richtung „Aufstieg durch Bildung“ als in Richtung Abstieg vom Gymnasium zur Realschule und von dieser zur Hauptschule (Mauthe/Rösner 1998). Auch ein zweigliedriges Schulsystem (bzw. ein dreigliedriges System, wenn man gesonderte Schulen für Lernbehinderte einbezieht) bleibt ein $T y$ pen-Schulsystem. Es bildet die historische Grundlage für das „Schisma“ (Baethge 2006) zwischen Allgemein- und Berufsbildung in der Form des dualen Systems. Wie Baethge herausgearbeitet hat, ist diese Organisationsform tief in der besonderen Industrieverfassung Deutschlands und der Verfassung der höheren Bildung (Gymnasien und Universitäten) als Ausbildungsstätten für den Nachwuchs an hohen Staatsbeamten verankert.
Wie tief das mehrgliedrige allgemeinbildende Schulsystem in der Gesellschaft der Bundesrepublik verwurzelt ist, merkt man immer dann, wenn es umfassender verändert werden soll. In Nordrhein-Westfalen (NRW) versucht die rot-grüne Minderheitsregierung derzeit (2011), $30 \%$ aller Haupt- und Realschulen und Gymnasien in „Gemeinschaftsschulen“ umzuwandeln, wenn dies auf der Ebene der Kommunen und der lokalen Elternschaften gewünscht wird. Der rechtliche Weg erfolgt über eine Experimentierklausel im Schulgesetz, das die integrierten "Gemeinschaftsschulen“ als Versuchsschulen erlaubt, aber insgesamt den Status des gegliederten Schulsystems schützt. Sogar in der Landesverfassung von NRW wird die „Gliederung des Schulwesens“ explizit genannt (vgl. Burger 2010). Man kann ziemlich sicher sein, dass ähnlich wie im Konflikt über die „Kooperative Schule“ in NRW vor drei Jahrzehnten und im Konflikt über die Verlängerung der Grundschule in Hamburg im Jahr 2009/10 das konservative Bündnis aus CDU, FDP, ständischen Lehrerverbänden der einzelnen Schulformen (unter Führung des Philologenverbandes), Elternverbänden und Bürgerinitiativen versuchen wird, das rot-grüne Schulprojekt mit Verweis auf Schulgesetz und Grundgesetz zu verhindern. Die Gliederung des Schulwesens, das Elternrecht auf Erziehung und das Recht der Eltern auf freie Wahl der Ausbildung ihrer Kinder bilden dabei eine Einheit: Nur weil es die Schulformen des gegliederten Schulwesens gibt, ist ihre Wahl nach der Primarausbildung zwingend. Gegenüber der staatlichen Grundschule gibt es nur das Recht auf Wahl einer Privatschule. Privatschulen werden in den letzten zehn Jahren in steigendem Maße frequentiert. Dabei ist der Anstieg bei den Kindern von Eltern mit Hochschulabschluss am stärksten (Lohmann et al. 2009).

Die Wahl weiterführender Schulformen im gegliederten Schulsystem ist nicht völlig frei, sondern hängt entscheidend von den Schulleistungen am Ende der Primarausbildung ab, die die Lehrer und Lehrerinnen feststellen und aus denen sie Empfehlungen für den Besuch weiterführender Schulen ableiten. Zwischen dem Schulwunsch der Eltern und der Schulempfehlung der Lehrer herrscht eine permanente Spannung. Die Eltern wünschen sich höhere Schulformen als die Lehrer bereit sind, gemäß der Schulleistungen zu empfehlen. Die Bundesländer regeln den Übergang unterschiedlich: In einigen Ländern sind die Schulleistungen entscheidend, in anderen der Elternwille (Arnold et al. 2010).

Wenn man sich die Wahl der Schulformen nach der Grundschule genauer ansieht, wird deutlich, weshalb mittlere und höhere Schichten ein starkes Interesse an der freien Wahl der Schulformen, insbesondere des Gymnasiums, äußern. Die obere Mittelschicht und die Oberschicht bzw. das sogenannte Bildungsbürgertum mögen zwar auch ein besonderes inhaltliches Interesse an den wissenschaftsnahen und kulturell anspruchsvollen Inhalten und Vermittlungsformen des Gymnasiums hegen. Zugleich ist jedoch offensichtlich, dass es handfeste materielle Interessen an der Wahl des Gymnasiums gibt, die das Bildungsbürgertum auch mit jenen mittleren und unteren Segmenten der Mittelschicht teilt, die dem Gymnasium kulturell nicht besonders nahe stehen dürften. Kinder aller mittleren und höheren Schichten werden bei deutlich niedrigeren Leistungen für das Gymnasium empfohlen als Kinder von Un- und Angelernten oder von Facharbeitern, die gewissermaßen ein LeistungsÜbersoll erbringen müssen, um eine Gymnasialempfehlung zu erlangen. Werden sie einmal empfohlen, realisieren ihre Eltern in geringerem Maße die Empfehlung der Lehrer als Eltern höherer Schichten. Bei Diskrepanzen zwischen der Schulempfehlung der Lehrer und dem Schulwunsch der Eltern setzen sich Eltern höherer Schichten häufiger über den Rat der Lehrer hinweg als Eltern aus unteren Schichten. Tillmann (2009) spricht deshalb zu Recht von einer „doppelten Benachteiligung“von Kindern unterer Schichten: Sie erzielen am Ende der Grundschulzeit niedrigere Leistungen als Kinder höherer Schichten (primärer Herkunftseffekt), und dann werden sie bei der Wahl der Schulformen noch zusätzlich benachteiligt (sekundärer Herkunftseffekt).

Bei gleicher Lesekompetenz am Ende der Grundschule und gleichen kognitiven Grundfähigkeiten haben Kinder aus der „oberen Dienstklasse“ (freie akademische Berufe, führende Angestellte und höhere Beamte, Unternehmer mit mehr als zehn Beschäftigten, Hochschullehrer) eine zweieinhalb mal so große Chance, für das Gymnasium (statt für die Realschule) empfohlen

$4 \quad$ Vgl. dazu den Überblick über die Situation in den Bundesländern bei Autorengruppe Bildungsberichterstattung 2010, S. $63 \mathrm{f}$. 
zu werden, als Kinder von Facharbeitern und Arbeitern mit Leitungsaufgaben. Bei der „unteren Dienstklasse“ (Angehörige der Semiprofessionen und des mittleren Managements, Beamte im mittleren u. gehobenen Dienst, nicht-manuell tätige technische Angestellte) liegt der Chancenvorteil beim 1,7-Fachen, bei kleinen Selbstständigen (unter zehn Beschäftigte) beim 1,5-Fachen (Bos et al. 2004, S. 213).

Wohlgemerkt: Die für alle Schichten gleichgesetzte Lesekompetenz und kognitive Grundfähigkeit am Ende der Grundschulzeit sind Leistungsmerkmale. Krasser lässt sich die Verletzung des Leistungsprinzips an dieser Stelle des Übergangs zu weiterführenden Schulen kaum ausdrücken. Man kann sagen, dass die Verknüpfung des Typenschulsystems mit der freien Wahl der Ausbildung seitens der Eltern eine sehr wirksame (weil rechtlich tief verankerte) Form der unauffälligen Dauer-Subvention des Nachwuchses höherer und mittlerer Schichten darstellt. Sie schöpfen völlig legal pure Statusvorteile im öffentlich finanzierten Bildungssystem ab und setzen allen Versuchen, dies einzuschränken, massiven Widerstand entgegen. Es mag sein, dass „das Bildungsbürgertum“ oder die obere Mittelschicht und die Oberschicht als Hauptprofiteure der institutionellen Regelungen auch die politischen Wortführer des Widerstands gegen umfassende Schulreformen sind. Aber wie das Beispiel des Volksentscheids gegen die Schulreform in Hamburg zeigt, kamen die hohen absoluten Zahlen der Reformgegner vermutlich auch dadurch zustande, dass ebenfalls untere Mittelschichten sich für das Gymnasium und gegen die Verlängerung der Grundschulzeit einsetzten (vgl. dazu genauer Draheim et al. 2010, S. 353ff.). Ein ganz anderes Problem stellt die geringe Mobilisierung der Arbeiterschaft bei einer Reform dar, die ihnen zugute kommen soll.

\subsection{DIE DAUER DER PRIMAR- AUSBILDUNG}

Neben der Koppelung von Elternwahlrecht und Typenschulsystem in der Sekundarstufe I stellt die Dauer der Primarausbildung ein weiteres Strukturmerkmal des deutschen Schulsystems dar, das die Entstehung herkunftsbedingter Ungleichheiten begünstigt. Konservative Schulpolitik bestand in Deutschland immer schon darin, die Verlängerung der Primarschulzeit zu verhindern. Nach Einführung der obligatorischen vierjährigen Grundschule im Jahr 1920 versuchten bürgerliche Schichten, ihre Kinder durch Nutzung von Ausnahmeregelungen von der vierjährigen Grundschule fernzuhalten und die beschlossene Auflösung der privaten dreijährigen Grundschulen hinauszuzögern. Sonderregelungen sollten es besonders fähigen Kindern erlauben, schon nach drei Jahren staatlicher Grundschule auf das Gymnasium überzuwechseln (Herrlitz et al. 2009, S.123f.).

Im Gegensatz dazu hat linke Schulpolitik immer auf eine Verlängerung der Primarschulzeit von vier auf (meist) sechs Jahre gedrängt und im Konzept der integrierten Gesamtschule die gemeinsame Grundbildung auf zehn Jahre ausgedehnt. Als maßgebliche Gründe für die Verlängerung der Grundschulzeit auf sechs Jahre wurden genannt: mehr Zeit für gemeinsames, Schüler unterschiedlicher Herkünfte integrierendes Lernen; mehr Zeit für kompensatorisches Lernen leistungsschwacher Schüler und Schülerinnen und für eine bessere Vorbereitung der Wahl einer weiterführenden Schulform. Im „Hamburger Schulkonflikt" beharrte die konservative Bürgerinitiative „Wir wollen lernen“ nicht nur auf dem Recht der Eltern auf freie Wahl der Schulformen, sondern auch auf der Beibehaltung der vierjährigen Grundschule. Der Bürgerinitiative war es ziemlich egal, was mit den Schulformen der Sekundarstufe außer dem Gymnasium passierte. Es musste nur die Wahlmöglichkeit „Gymnasium oder irgendeine andere Schulform“ erhalten bleiben, und das Gymnasium durfte nicht um die Klassen fünf und sechs verkürzt werden. Je früher aber Bildungswahlen der Eltern in einem gegliederten Schulsystem die weitere Schullaufbahn bestimmen und je häufiger sie stattfinden, desto stärker wird der Einfluss der sozialen Herkunft auf den Schulerfolg sein (Baumert/Schümer 2001, S. 354). Insofern ist das eigensinnige und nicht hinnehmbare Beharren des Bürgertums auf der unverkürzten Länge der gymnasialen Ausbildung nicht nur ein Hebel, um das Gymnasium als besondere Schulform zu erhalten. Vielmehr wird so dem gesamten Bildungssystem aufgezwungen, dass mit der vierten Jahrgangsstufe die Phase endet, in der gleiche Startbedingungen für den anschließenden Bildungswettbewerb geschaffen werden könnten. Das ist eine ausgesprochen restriktive und billige Lösung.
Dies gilt umso mehr, wenn man die Kompetenzen einbezieht, über die jedes Gesellschaftsmitglied zur politischen und sozialen Teilhabe verfügen soll. Für den engeren Bereich des politischen Lernens ist völlig klar, dass es nicht im Grundschulalter abgeschlossen sein kann. Wegen seiner Bindung an die längerfristige moralische und kognitive Entwicklung führt es erst in der mittleren bis späten Adoleszenz zu einem stabilen und ausgereiften Niveau, d.h. frühestens am Ende der Sekundarstufe I. Im herrschenden Typenschulsystem sind die Unterschiede der politischen Kompetenzen zwischen den Schulformen und den unterschiedlichen sozialen Herkunftsbedingungen beträchtlich (vgl. Hopf/Hopf 1997, S. 181ff.). Auch bei den sprachlichen, mathematischen und naturwissenschaftlichen Kompetenzen sind in Deutschland die Streuungen um die Mittelwerte am Ende der Sekundarstufe I stark (Deutsches PISA-Konsortium 2001, S. 105ff., S. 173ff.). Sie sind deutlich höher als am Ende der Grundschulzeit (vgl. Bos et al. 2007, S. 153). Die Kompetenzunterschiede der 15-Jährigen nach Schichtzugehörigkeit sind im internationalen Vergleich besonders ausgeprägt (Deutsches PISA-Konsortium 2001, S. 386ff.; Ehmke/ Baumert 2007, S. 315ff.).

Die Unterschiede zwischen der Schichtabhängigkeit des Bildungserfolgs am Ende der Grundschule und am Ende der Sekundarstufe I sind ein Indiz dafür, dass in Deutschland die historisch gegebene Schulorganisation die Startchancengleichheit für den Bildungswettbewerb nach der Grundschule nicht vollständig ermöglicht, dagegen gleiche Basiskompetenzen für gesellschaftliche Teilhabe sogar massiv behindert. Wenn man immer nur die fehlende Leistungsgerechtigkeit an den Übergängen des deutschen Bildungssystems kritisiert und den chancengleichen „Aufstieg durch Bildung" fordert, bleibt man in der Konkurrenzlogik fixiert und übersieht die viel weitergehende Beschränkung, die das ungleiche Bildungssystem für die gesellschaftliche Teilhabe darstellt (vgl. hierzu auch Becker in diesem Heft). Durch das Festhalten an der Eigenständigkeit des Gymnasiums und an der nur vierjährigen Grundschule spaltet das Bürgertum die Gesellschaft. Dabei sei dahingestellt, ob gerade jetzt die abstiegsbedrohten Teile der Mittelschicht (vgl. hierzu Vogel 2009) und die aufstiegszuversichtlichen unteren Teile der Mittelschicht eine besonders aggressi- 
ve Koalition zugunsten des Gymnasiums und der herkömmlichen Schulstruktur eingehen. Diese schulpolitische Koalition unterschiedlicher Teile der Mittel- und Oberschicht besteht in Deutschland schon seit Langem.

\section{1}

\section{Was ist zu tun?}

Unabhängig von Verbesserungen des Unterrichts und der Lehrerbildung, des Ausbaus von Ganztagsschulen und der Integration von Haupt- und Realschulen stellen sich innerhalb des bestehenden Systems folgende Aufgaben:

(1) Die Vorschulerziehung sollte weiter ausgebaut und die Grundschule in die Lage versetzt werden, die noch verbleibenden Kompetenzunterschiede am Ende der vierten Klasse anzugleichen. Die große Bedeutung der vorschulischen Bildung für spätere Lernprozesse wird inzwischen allgemein anerkannt. Dabei geraten zunehmend auch die sehr frühen Bildungsprozesse noch vor dem Kindergartenalter in den Blick (vgl. Heckman/ Masterov 2007). Eine flächendeckend eingeführte vorschulische Bildung hilft überdies, weitere sozial- und beschäftigungspolitische Probleme zu lösen, z.B. die Steigerung der in Deutschland immer noch niedrigen Frauenerwerbstätigkeit, die mit betrieblichen Veränderungen zur Vereinbarkeit von Familie und Beruf abgestützt werden muss, sowie die Stützung Alleinerziehender (meist Mütter), die ein besonders hohes Armutsrisiko tragen.
(2) Es sollte der Übergang in das Typenschulsystem so umgestaltet werden, dass das Recht der Eltern auf freie Schulwahl durch Leistungen fundiert wird und nicht die mittleren und höheren Schichten begünstigt. $\mathrm{Zu}$ denken, mit wenigen Erlassen zur bürokratischen Regelung des Übergangs das schichtspezifische Übergangsproblem lösen zu können, ist naiv. Ein zwar aufwendiges, aber effektives Alternativmodell, das Herkunftseffekte abschwächt, stellt das schweizerische Übergangsverfahren im Kanton Freiburg dar (vgl. Baeriswyl et al. 2006). Hier wird die Leistungsrückmeldung am Ende der sechsjährigen Primarausbildung verbreitert (Noten und Testergebnisse). Die Eltern werden in einem intensiven Beratungsprozess unter Einschluss von Vertretern der aufnehmenden Sekundarschule einbezogen. Daran schließt sich eine halbjährige Erprobungsphase im gewählten Bildungsgang an, der noch gewechselt werden kann.

(3) Weitere, über das bestehende Schulsystem hinausgehende Maßnahmen wären zum einen, die Grundschulausbildung zu verlängern, und zweitens, das Ziel einer zehnjährigen Grundbildung für gleiche gesellschaftliche Teilhabe anzustreben. Die Definition von „Basiskompetenzen“, die PISA im Anschluss an die OECD formulierte (siehe oben), ist dabei ein sehr sinnvoller Ansatzpunkt. Jedoch sind gegenüber den eng und konventionell definierten Maßen der Kompetenz in den internationalen Schulleistungsstudien (meistens Lesekompetenz, Mathematik, Naturwissenschaft) die „weicheren“ Kompetenzen in politischer Bildung, Menschenrechtsbildung oder musischer Bildung stärker zu gewichten, weil durch sie die außerberufliche gesellschaftliche Teilhabe befördert wird.

(4) Förderlich wäre ferner die Integration aller Schulformen über bisherige Ansätze hinaus. Das meint vor allem den Ausbau von Gesamtschulen. Dafür setzt das rotgrüne Projekt in Nordrhein-Westfalen, schrittweise eine "Gemeinschaftsschule“ unter Einschluss der Gymnasien einzuführen, einen gewichtigen Meilenstein.

(5) Und schließlich muss die Forderung nach gleicher gesellschaftlicher Teilhabe mit dem Ziel der „Integration“von Migranten und ihren Kindern verknüpft werden. Es ist erstaunlich, wie sehr sich die öffentliche Diskussion über Chancengleichheit auf die Situation von Migranten konzentriert und wie wenig gesehen wird, dass ein maßgeblicher Teil der Benachteiligung von Migranten ihrem Schichtungsstatus zuzuschreiben ist. Kein Mensch käme auf die Idee, Kinder von deutschen un- und angelernten Arbeitern in dem Sinne „kulturell integrieren“ zu wollen wie Kinder von Migranten. Aber faktisch sind auch sie im Bildungssystem nicht integriert. Wenn man einen erweiterten, gemeinsamen Integrationsbegriff entwickelt, erscheint die Trennung aller Kinder und Jugendlichen durch das herkömmliche Schultypensystem in Deutschland überholt. Künftige Schülergenerationen werden sich vielleicht fragen: Wie kamen die Befürworter der einzelnen Schultypen überhaupt auf die Idee, die Schüler und Schülerinnen so einzusortieren und voneinander zu trennen? Bis dahin ist es allerdings noch ein langer Weg. 
Allmendinger, J./Leibfried, S. (2002): Bildungsarmut im Sozialstaat, in: Burkart, G./Wolf, J. (Hrsg.): Lebenszeiten. Erkundungen zur Soziologie der Generationen, Opladen, S. 287-315

Arnold, K.-H./Bos, W./Richert, P./Stubbe, T. C. (2010): Der Übergang von der Grundschule in die Sekundarstufe: Schullaufbahnpräferenzen von Lehrkräften und Eltern im Ländervergleich, in: Bos, W./Hornberg, S./Arnold, K.-H./Faust, G./Fried, L./Lankes, E.-M./Schwippert, K./ Tarelli, I./Valtin, R. (Hrsg.): IGLU 2006 - die Grundschule auf dem Prüfstand. Vertiefende Analysen zu Rahmenbedingungen schulischen Lernens, Münster/New York/München/Berlin, S. 13-32

Autorengruppe Bildungsberichterstattung (2010): Bildung in Deutschland 2010. Ein indikatorengestützter Bericht mit einer Analyse zu Perspektiven des Bildungswesens im demografischen Wandel, Bielefeld Baeriswyl, F./Wandeler, C./Trautwein, U./Oswald, K. (2006): Leistungstest, Offenheit von Bildungsgängen und obligatorische Beratung der Eltern. Reduziert das Deutschfreiburger Übergangsmodell die Effekte des sozialen Hintergrunds bei Übergangsentscheidungen?, in: Zeitschrift für Erziehungswissenschaft 9 (3), S. 373-392

Baethge, M. (2006): Das deutsche Bildungs-Schisma: Welche Probleme ein vorindustrielles Bildungssystem in einer nachindustriellen Gesellschaft hat, in: SOFI-Mitteilungen (34), Dezember 2006, S. 13-27 Baumert, J./Schümer, G. (2001): Familiäre Lebensverhältnisse, Bildungsbeteiligung und Kompetenzerwerb, in: Deutsches PISAKonsortium (Hrsg.): PISA 2000. Basiskompetenzen von Schülerinnen und Schülern im internationalen Vergleich, Opladen S. 323-407 Bos, W. /Hornberg, S./Arnold, K.H./Faust, G./Fried, L./Lankes, E.-M./Schwippert, K./Valentin, R. (Hrsg.) (2007): IGLU 2006. Lesekompetenzen von Grundschulkindern in Deutschland im internationalen Vergleich, Münster/New York/München/Berlin Bos, W./Schwippert, K./Stubbe, T. C. (2007): Die Koppelung von sozialer Herkunft und Schülerleistung im internationalen Vergleich, in: Bos, W. et al. (Hrsg.): IGLU 2006, a.a.O., S. 225-247

Bos, W./Valtin, R./Hornberg, S./Buddeberg, I./Goy, M./Voss, A. (2007): Internationaler Vergleich 2006: Lesekompetenzen von Schülerinnen und Schülern am Ende der vierten Jahrgangsstufe, in: Bos, W. et al. (Hrsg. ): IGLU 2006, a.a.O., S. 109-160

Bos, W./Voss, A./Lankes, E.-M./Schwippert, K./Thiel, O./Valtin, R. (2004): Schullaufbahnempfehlungen von Lehrkräften für Kinder am Ende der vierten Jahrgangsstufe, in: Bos, W./Lankes, E.-M./Prenzel, M./Schwippert, K./Valtin, R./Walther, G. (Hrsg.): IGLU. Einige Länder der Bundesrepublik Deutschland im nationalen und internationalen Vergleich, Münster/New York/München/Berlin, S. 191-222 Burger, R. (2010): Konsens oder Nonsens? FAZ vom 23. September, S. 4
Deutsches PISA-Konsortium (Hrsg.) (2001): PISA 2000.

Basiskompetenzen von Schülerinnen und Schülern im internationalen Vergleich, Opladen

Draheim, S./Krause, A./Reitz, T. (2010): Von Chancen und Statuskämpfen. Klassenverhältnisse in der Erziehungsgesellschaft, in: Prokla 160, 40 (3), S. 341-360

Ehmke, T./Baumert, J. (2007): Familiäre Lebensverhältnisse und Kompetenzerwerb, in: PISA-Konsortium Deutschland (Hrsg.): PISA 2006. Die Ergebnisse der dritten internationalen Vergleichsstudie, Münster/New York/München/Berlin, S. 309-335

Heckman, J. J./Masterov, D. V. (2007): The productivity argument for investing in young children, http://jenni.uchicago.edu/ human-inequality/papers/Heckman_final_all_wp_2007-03-22c_jsb.pdf, Zugriff am 7.12.2010

Herrlitz, H.-G./Hopf, W./Titze, H./Cloer, E. (2009):

Deutsche Schulgeschichte von 1800 bis zur Gegenwart. Eine Einführung, Weinheim/München

Hopf, W. (2010): Freiheit - Leistung - Ungleichheit. Bildung und soziale Herkunft in Deutschland, Weinheim/München

Hopf, C./Hopf, W. (1997): Familie, Persönlichkeit, Politik. Einführung in die politische Sozialisation, Weinheim/München Kronauer, M. (2010): Exklusion. Die Gefährdung des Sozialen im hoch entwickelten Kapitalismus, 2. Aufl., Frankfurt a.M./New York Lohmann, H./Spieß, C. K./Feldhaus, C. (2009): Der Trend zur Privatschule geht an bildungsfernen Eltern vorbei, in: DIW Wochenbericht 76 (38), S. 640-649

Mauthe, A./Rösner, E. (1998): Schulstruktur und Durchlässigkeit, in: Rolff, H.-G. et al. (Hrsg.): Jahrbuch der Schulentwicklung, Bd. 10, Weinheim/Basel, S. 87-125

Pollak, R. (2010): Kaum Bewegung, viel Ungleichheit. Eine Studie zu sozialem Auf- und Abstieg in Deutschland, Schriften zu Wirtschaft und Soziales, Bd. 5, hrsg. von der Heinrich-Böll-Stiftung, Berlin Streckeisen, U./Hänzi, D./Hungerbühler, A. (2007): Fördern und Auslesen. Deutungsmuster von Lehrpersonen zu einem beruflichen Dilemma, Wiesbaden

Tillmann, K.-J. (2009): Sechsjährige Primarschule in Hamburg: Empirische Befunde und pädagogische Bewertungen, in: Hamburg macht Schule. Zeitschrift für Hamburger Lehrkräfte und Elternräte, Sonderheft 2009, S. 10-29

Välijärvi, J. (2008): Chancengleichheit - Voraussetzung für hohe Qualität, in: Sarjala, J./Häkli, E. (Hrsg.): Jenseits von PISA. Finnlands Schulsystem und seine neuesten Entwicklungen, Berlin, S. 79-98 Vogel, B. (2009): Wohlstandskonflikte. Soziale Fragen, die aus der Mitte kommen, Hamburg 